\title{
Plastic disconnection of storey masses for seismic stress control
}

\author{
M. C. Porcu \\ Department of Mechanical, Chemical and Materials Engineering, \\ University of Cagliari, Italy
}

\begin{abstract}
The paper detects the role of affecting parameters in the performance of a stress control technique based on disconnecting seismic masses during strong earthquakes. Such a technique assumes that rigid-plastic connectors are inserted between structural and non-structural floor masses to detach portions of active mass as soon as a pre-set level of horizontal load is reached. By means of a wide numerical investigation on linear and non-linear single-storey frames acted upon by different strong recorded ground motions, the present paper highlights the ranges within which the governing parameters should be set to make the stress control effective. In view of a practical application of the considered technique, a simple procedure is also provided to derive the stress reduction from the EC8 design response spectrum for given values of the key parameters.

Keywords: plastic disconnection of floor mass, seismic vibration control, inertia limiters, rigid-plastic connectors, prediction of stress reduction.
\end{abstract}

\section{Introduction}

Reducing seismic stress on buildings is of great concern for civil engineers. A classical way to achieve this purpose is by designing ductile structures, as prescribed by seismic codes. Well-established techniques are also based on energy dissipaters inside the structure [1] or isolators at its base [2]. The principal feature of base-isolation techniques is, in fact, that of reducing stiffness, which is expected to reduce earthquake-induced accelerations. Alternative approaches $[3,4]$ are based on reducing the active mass of buildings. Among them, the plastic disconnection of floor masses method (PDFMM), investigated in [5-8], assumes that rigid-perfectly-plastic devices (very stiff in 
the elastic range, in practice) are interposed between structural and non-structural floor masses. Under strong earthquakes such connectors may plastically yield and thus disconnect portions of seismic mass. This is expected to reduce inertial loads on storeys and therefore seismic stress on columns.

The effectiveness of the PDFMM can be assessed by means of two quantities: the stress reduction that may be achieved and the peak relative displacement of the disconnected mass. The value of these quantities depends on different interrelated parameters: the portion of mass disconnected, the yield limit of connectors, the natural period and damping ratio of the system and the earthquake acting on it. By broadening the contribution given in [8], the present paper assesses the role of key parameters and identifies the ranges within which they should be set to make the PDFMM effective. To this end, a wide-ranging numerical investigation was performed with different recorded earthquakes and tens of linear and nonlinear systems. Results highlight a strong influence of both percentage of mass disconnected and connector yield limit. A slight influence of damping ratio is found instead. Whilst a more marked dependence from the natural period of the corresponding elastic system is generally observed. Quite small relative displacements are generally found even for high stress reduction.

An approximate method for estimating the stress reduction from the earthquake elastic response spectrum is provided in [6]. Based on it, the present paper derives a simple procedure to obtain the stress reduction from the Eurocode 8 (EC8) design response spectrum. This procedure could be associated to the one derived in [7] that estimates the peak displacement of disconnected masses from the earthquake rigid-plastic spectrum, a single curve diagram introduced in [9].

Although further investigations are certainly required to definitely assess the effectiveness of the PDFMM, the results presented in this paper highlight the potential of this technique of stress control, thereby encouraging further in-depth investigations with a view to design details for its practical application.

\section{Analytical model}

Reference to a shear-type single-storey frame of mass $m$, lateral stiffness $k$ and damping ratio $\xi$ will be done here (fig. 1). Frame floor mass $m$ is assumed to be divided into two parts, say an underlying mass $m_{1}$ and an upper mass $m_{2}$ (so that $m_{1}+m_{2}=m$ ), joined together through a set of inelastic connectors. For practical purposes, connectors are assumed to be as stiff as possible in the elastic range (rigid-plastic connectors). They are all taken into account through an equivalent rigid-plastic connector, the behaviour of which is depicted in fig. 1 , where $F_{y}$ denotes the absolute value of the reaction $F_{R}$ at yield.

The frame in fig. 1 is a two degree-of-freedom nonlinear system. Under a given ground acceleration $\ddot{u}_{g}(t)$, its motion equations can be written as (cf. [58]): 

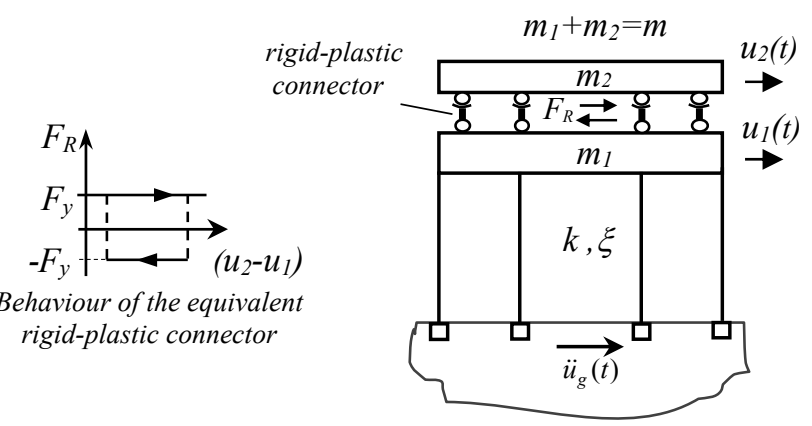

Figure 1: Shear-type frame with rigid-plastic connectors between floor masses.

\section{Elastic range}

$$
\text { if } \begin{aligned}
\left|\ddot{u}_{2}^{t}\right|<a_{y} \quad \text { or if } & \left|\ddot{u}_{2}^{t}\right|=a_{y} \text { and } \operatorname{sign}\left(\dot{u}_{2}\right) \neq \operatorname{sign}\left(u_{2}\right) \\
& \left\{\begin{array}{l}
\ddot{u}_{1}+\frac{4 \pi \xi}{T} \dot{u}_{1}+\frac{4 \pi^{2}}{T^{2}} u_{1}=-\ddot{u}_{g}(t) \\
u_{2}=u_{1}
\end{array}\right.
\end{aligned}
$$

Plastic range

$$
\begin{aligned}
& \text { if }\left|\ddot{u}_{2}^{t}\right|=a_{y} \quad \text { and } \operatorname{sign}\left(\dot{u}_{2}\right)=\operatorname{sign}\left(u_{2}\right) \\
& \left\{\begin{array}{l}
\left(1-\rho_{m}\right) \ddot{u}_{1}+\frac{4 \pi \xi}{T} \dot{u}_{1}+\frac{4 \pi^{2}}{T^{2}} u_{1}-\operatorname{sign}\left(u_{2}-u_{1}\right) a_{y} \rho_{m}=\left(\rho_{m}-1\right) \ddot{u}_{g}(t) \\
\ddot{u}_{2}+\operatorname{sign}\left(u_{2}-u_{1}\right) a_{y}=-\ddot{u}_{g}(t)
\end{array}\right.
\end{aligned}
$$

Here $u_{1}=u_{1}(t), u_{2}=u_{2}(t) ; \dot{u}_{1}=\dot{u}_{1}(t), \dot{u}_{2}=\dot{u}_{2}(t) ; \ddot{u}_{1}=\ddot{u}_{1}(t), \ddot{u}_{2}=\ddot{u}_{2}(t)$ are displacement, velocity and acceleration of masses $m_{1}$ and $m_{2}$, respectively (all relative to the ground). On the contrary, $\ddot{u}_{2}^{t}(t)=\ddot{u}_{2}(t)+\ddot{u}_{g}(t)$ denotes the total acceleration (inertial) of mass $m_{2}$. Parameter $a_{y}$ is defined as $a_{y}=F_{y} / m_{2}$ and represents the absolute value of the total acceleration of mass $m_{2}$ at yield (yield acceleration), while $\rho_{m}=m_{2} / m$ is the mass ratio quantifying the percentage of disconnected mass. The following dimensionless parameter, referred to as the yield ratio, may be more usefully introduced in place of $a_{y}$ :

$$
\rho_{y}=\frac{a_{y}}{\ddot{u}_{E}^{t}}
$$


Quantity $\ddot{u}_{E}^{t}$ appearing in eqn (4) denotes the peak total acceleration of the equivalent elastic frame of mass $m$, stiffness $k$ and damping $\xi$. It could be also taken here as the peak total acceleration that mass $m_{2}$ would reach under the given earthquake should connectors be perfectly rigid. The yield ratio $\rho_{y}$ may vary within the range $0 \leq \rho_{y} \leq 1$, the end values of which meaning that mass $m_{2}$ is completely detached from mass $m_{1}\left(\rho_{y}=0\right)$ or that mass $m_{2}$ is rigidly connected to mass $m_{1}\left(\rho_{y}=1\right)$ respectively. Of course, both of these extreme situations have no practical interest.

\section{Role of key parameters on stress control}

To investigate the effectiveness of the PDFMM, the following two quantities may be considered, see [6-8]:

$$
\begin{gathered}
\Delta_{S}=\Delta_{S}\left(\rho_{m}, \rho_{y}, \xi, T\right)=\frac{u_{1 \max }}{u_{E}}-1 \\
u_{21 \text { max }}=u_{21 \max }\left(\rho_{m}, \rho_{y}, \xi, T\right)=\left|\max \left[u_{2}(t)-u_{1}(t)\right]\right|
\end{gathered}
$$

Here $u_{1 \max }=\max \left|u_{1}(t)\right|$ denotes the peak displacement of mass $m_{1}$, as obtained by solving eqns (3a)-(3b), while $u_{E}$ is the peak displacement of the equivalent elastic frame of mass $m$, stiffness $k$ and damping $\xi$. Ratio $\Delta_{S}$ may be referred to as the stress reduction factor, while $u_{21 \max }$ is the peak relative displacement of mass $m_{2}$ with respect to mass $m_{1}$. Both $\Delta_{S}$ and $u_{21 \max }$ depend on four parameters: $\rho_{m}, \rho_{y}, \xi$ and $T$.

By referring to the recorded strong earthquakes listed in Table 1 and to linear and nonlinear systems, an in-depth numerical investigation was performed to obtain the values of both $\Delta_{S}$ and $u_{21 \max }$ for different values of the key parameters. Some results are given in figs 2-5. Diagrams of fig. 2(a) show that to obtain an effective and realistic stress reduction, $\rho_{m}$ should be set between 0.2 and 0.5 ; there is no practical interest in further increasing its value due to a sort of "knee" exhibited by all the curves. Fig. 3(a) shows that a suitable range for $\rho_{y}$ may be $0.05 \leq \rho_{y} \leq 0.2$. In particular, fig. 3(a) shows that a stress reduction even up to $50 \%$ can be obtained in this range, provided that one third of the mass is disconnected. From fig. 3(a) it can be also inferred that the stress reduction may even overcome the $60 \%$ when one half of the mass is disconnected.

Whatever the earthquake, however, there is no convenience in setting the value of $\rho_{y}$ out of the above mentioned range. Apart from practical reasons that would dissuade this choice, a value of $\rho_{y}$ lower than 0.05 would actually imply 
an increase in relative displacements (due to the drop in plastic energy dissipation) against a slight increase in stress reduction. On the other hand, values of $\rho_{y}$ higher than 0.2 would drastically reduce the beneficial effects of mass disconnection.

Table 1: Ground motions considered in the investigation.

\begin{tabular}{|c|c|c|}
\hline Code & Recorded earthquake & PGA (m/s $\mathbf{~}$ ) \\
\hline E1 & Parkfield (California), 90, 2004 & 5.80 \\
\hline E2 & Chi-Chi (Taiwan), CHY041N, 1999 & 6.30 \\
\hline E3 & El Centro (California), 1940 & 3.20 \\
\hline E4 & Kocaeli (Turkey), ATS000, 1999 & 2.49 \\
\hline E5 & Irpinia (Italy), A-STU270, 1980 & 3.51 \\
\hline
\end{tabular}

Even if a slightly larger stress reduction is found for lower values of $\xi$, this parameter have generally a quite small effect on the effectiveness of the method, see fig. 4(a). On the contrary, a much greater influence of the natural period $T$ on $\Delta_{S}$ is highlighted by Fig. 5(a). It can be noted, finally, that in all of the instances considered rather low values of $u_{21 \max }$ are found, figs 2(b), 3(b), 4(b), 5(b).
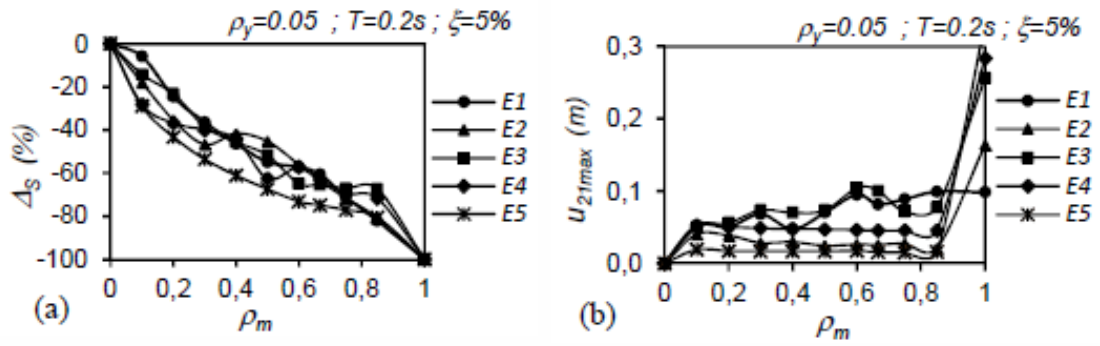

Figure 2: Impact of $\rho_{m}$ on (a) stress reduction; (b) peak relative displacement.
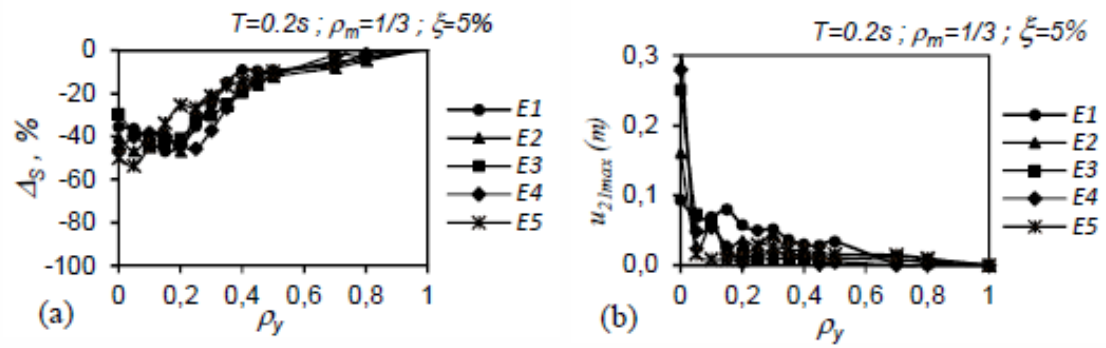

Figure 3: Impact of $\rho_{y}$ on (a) stress reduction; (b) peak relative displacement. 

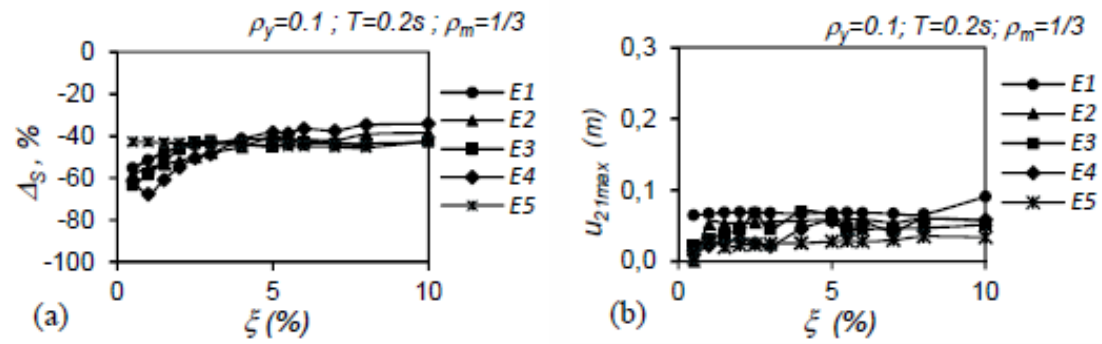

Figure 4: Impact of $\xi$ on (a) stress reduction; (b) peak relative displacement.
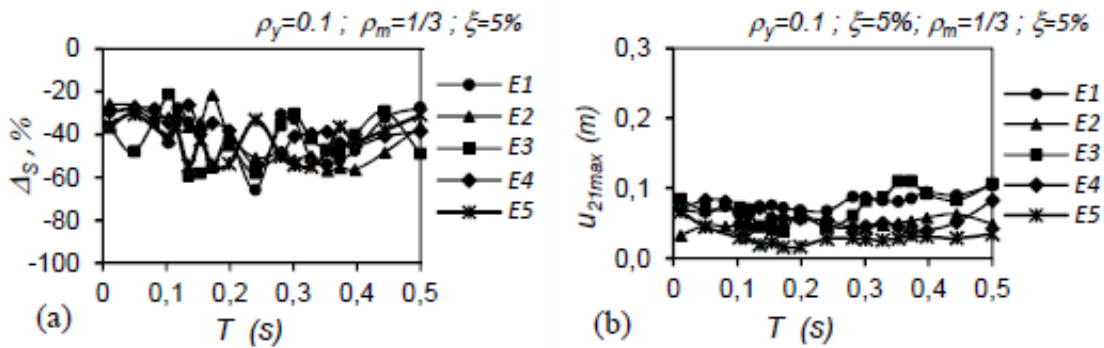

Figure 5: Impact of $T$ on (a) stress reduction; (b) peak relative displacement.

\section{Stress reduction from the EC8 design spectrum}

A good estimate of the stress reduction factor may be obtained from the following formula, derived and assessed in [6]:

$$
\Delta_{S} \cong \sqrt{\left(\frac{u_{1 E}}{u_{E}}\right)^{2}+\frac{\rho_{y}^{2} \rho_{m}^{2}}{\left(1-\rho_{m}\right)^{2}}\left(1+e^{\frac{-\pi \xi}{\sqrt{\rho_{m}-\xi^{2}}}}\right)^{2}}-1
$$

Here $u_{1 E}=u_{1 E}\left(T_{1}, \xi_{1}\right)$ denotes the peak displacement of a linear elastic system with natural period $T_{1}=2 \pi \sqrt{m_{1} / k}=T \sqrt{\left(1-\rho_{m}\right)}$ and damping ratio $\xi_{1}=\xi / \sqrt{\left(1-\rho_{m}\right)}$, whilst $u_{E}=u_{E}(T, \xi)$ is the peak elastic displacement of a system with natural period $T$ and damping ratio $\xi$. Of course, both the values of $u_{1 E}$ and $u_{E}$ may be taken from the elastic response spectrum.

Eqn (7) will be herein exploited to estimate the stress reduction from the design response spectrum. To this purpose, the pseudo peak accelerations 
$\ddot{u}_{1 E}^{*}=\ddot{u}_{1 E}^{*}\left(T_{1}, \xi_{1}\right) \cong\left(k / m_{1}\right) u_{1 E}$ and $\ddot{u}_{E}^{*}=\ddot{u}_{E}^{*}(T, \xi) \cong(k / m) u_{E}$ are considered. As a function of the ratio $R_{A}=\ddot{u}_{1 E}^{*} / \ddot{u}_{E}^{*}$, eqn (7) can be put as:

$$
\Delta_{S} \cong \sqrt{\left(1-\rho_{m}\right)^{2} R_{A}^{2}+\frac{\rho_{y}^{2} \rho_{m}^{2}}{\left(1-\rho_{m}\right)^{2}}\left(1+e^{\frac{-\pi \xi}{\sqrt{\rho_{m}-\xi^{2}}}}\right)^{2}}-1
$$

With reference to EC8 [10], the spectral accelerations $S_{E}\left(T_{1}, \xi_{1}\right)$ and $S_{E}(T, \xi)$ are now taken into consideration. As a function of them, the ratio $R_{A}=S_{E}\left(T_{1}, \xi_{1}\right) / S_{E}(T, \xi)$ can be obtained as:

for $0 \leq T \leq T_{B}$

$$
R_{A}=\frac{\sqrt{(5+\xi)}}{\sqrt{\left(5+\frac{\xi}{\sqrt{\left(1-\rho_{m}\right)}}\right)}} \frac{\frac{T \sqrt{\left(1-\rho_{m}\right)}}{T_{B}}+\frac{\sqrt{\left(5+\frac{\xi}{\sqrt{\left(1-\rho_{m}\right)}}\right)}}{F_{o} \sqrt{10}}\left(1-\frac{T \sqrt{\left(1-\rho_{m}\right)}}{T_{B}}\right)}{\left[\frac{T}{T_{B}}+\frac{\sqrt{(5+\xi)}}{F_{o} \sqrt{10}}\left(1-\frac{T}{T_{B}}\right)\right]}
$$

for $T_{B} \leq T \leq \frac{T_{B}}{\sqrt{\left(1-\rho_{m}\right)}}$

$$
\begin{aligned}
& R_{A}=\frac{\sqrt{(5+\xi)}}{\sqrt{\left(5+\frac{\xi}{\sqrt{\left(1-\rho_{m}\right)}}\right)}}\left[\frac{T \sqrt{\left(1-\rho_{m}\right)}}{T_{B}}+\frac{\sqrt{\left(5+\frac{\xi}{\sqrt{\left(1-\rho_{m}\right)}}\right)}}{F_{o} \sqrt{10}}\left(1-\frac{T \sqrt{\left(1-\rho_{m}\right)}}{T_{B}}\right)\right] \\
& \text { for } \frac{T_{B}}{\sqrt{\left(1-\rho_{m}\right)}} \leq T \leq T_{C} \quad R_{A}=\frac{\sqrt{(5+\xi)}}{\sqrt{\left(5+\xi / \sqrt{\left(1-\rho_{m}\right)}\right)}} \\
& \text { for } T_{C} \leq T \leq \frac{T_{C}}{\sqrt{\left(1-\rho_{m}\right)}} \quad R_{A}=\frac{\sqrt{(5+\xi)}}{\sqrt{\left(5+\xi / \sqrt{\left(1-\rho_{m}\right)}\right)} \frac{T}{T_{C}}} \\
& \text { for } \frac{T_{C}}{\sqrt{\left(1-\rho_{m}\right)}} \leq T \leq T_{D} \quad R_{A}=\frac{\sqrt{(5+\xi)}}{\sqrt{\left(1-\rho_{m}\right)} \sqrt{\left(5+\xi / \sqrt{\left(1-\rho_{m}\right)}\right)}}
\end{aligned}
$$




$$
\begin{array}{cc}
\text { for } T_{D} \leq T \leq \frac{T_{D}}{\sqrt{\left(1-\rho_{m}\right)}} \quad R_{A}=\frac{\sqrt{(5+\xi)}}{\sqrt{\left(1-\rho_{m}\right)} \sqrt{\left(5+\xi / \sqrt{\left(1-\rho_{m}\right)}\right)}} \frac{T}{T_{D}} \\
\text { for } T \geq \frac{T_{D}}{\sqrt{\left(1-\rho_{m}\right)}} \quad R_{A}=\frac{\left(1-\rho_{m}\right) \sqrt{(5+\xi)}}{\sqrt{\left(5+\xi / \sqrt{\left(1-\rho_{m}\right)}\right)}}
\end{array}
$$

The value of $\xi$ should be introduced in percent into the above formulas.

Once that ratio $R_{A}$ is obtained from eqns (9)-(15) and put into eqn (8), the stress reduction relevant to the design earthquake may be derived. An instance of how $\Delta_{S}$ can be estimated from the EC8 design spectrum by means of eqn (8) and eqns (9)-(15), is provided in fig. 6 . It shows that a stress reduction between $40 \%$ and $60 \%$ may be attained for oscillators with natural period lower than $0.3 \mathrm{~s}$, provided that one half of the floor mass is disconnected and the connector yield limit is set as to have $\rho_{y} \leq 0.05$.

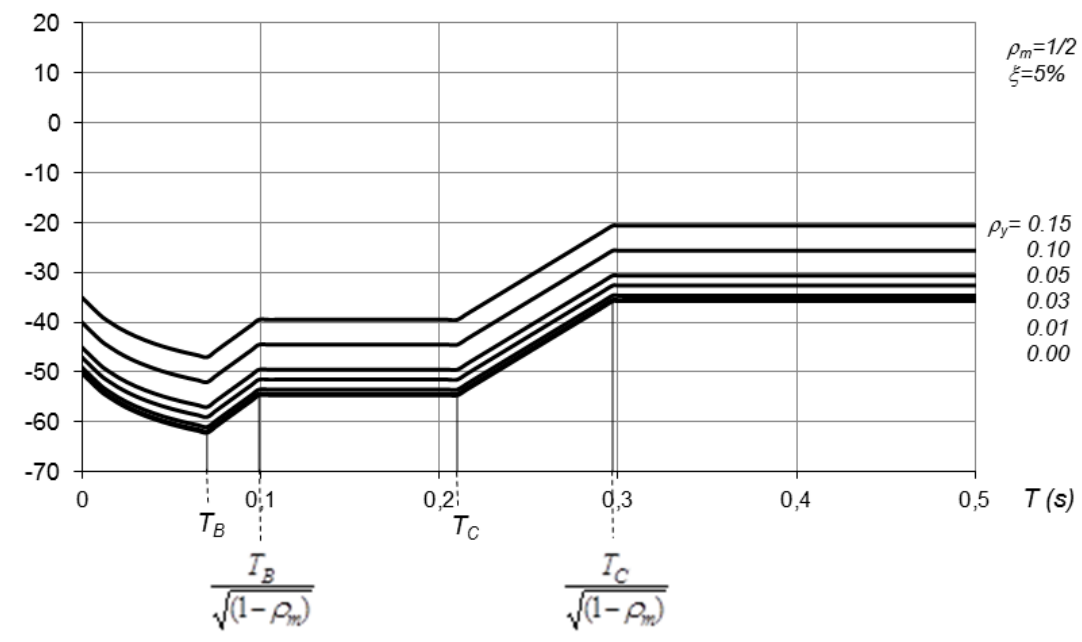

Figure 6: An instance of how stress reduction can be obtained from the EC8 elastic response spectrum by means of eqns (8)-(15).

\section{Conclusions}

The role of affecting parameters in the performance of the plastic disconnection of floor mass method for seismic control has been assessed in the paper. By means of a wide numerical investigation the paper shows that whatever the earthquake the technique considered may be effective when (i) a portion between 
one fifth and one half of the total floor mass is disconnected); (ii) the yield limit of connectors is set between $5 \%$ and $15 \%$ of the peak elastic force. Results also show that there is no convenience in further increasing the portion of disconnected mass or decreasing the yield limit. A slight influence of damping ratio was found, whilst a more marked dependence on the natural period was detected instead. For the purpose of practical application of the method, the paper provides some formulas for estimating the stress reduction directly from the EC8 design response spectrum for any given value of the key parameters.

\section{References}

[1] M.D. Symans, F.A. Charney, A.S. Whittaker, M.C. Constantinou, C.A. Kircher, M.W. Johnson, and R.J. McNamara, "Energy Dissipation Systems for Seismic Applications: Current Practice and Recent Developments", J Struct Eng, 134 (1), pp. 3-21, 2008.

[2] I.J. Buckle, R.L. Mayes, "Seismic isolation: history, application and performance - A world view", Earthq. Spectra, 6(2), pp. 161-201, 1990.

[3] R. Villaverde, "Roof Isolation System to Reduce the Seismic Response of Buildings: A Preliminary Assessment”, Earthq. Spectra, 14(3), pp. 521532, 1998.

[4] M. Ziyaeifar and H. Noguchi, "Partial Mass Isolation in Tall Buildings", Earthq. Eng. \& Struct. Dyn., 27, pp. 49-65, 1998.

[5] A. Paglietti and M.C. Porcu, "Controlling Dynamic Stress through Plastic Limiters", in Proc. 1st European Conference on Structural Control, A. Baratta \& J. Rodelar (eds), World Scientific Publishing, Singapore, 483490, 1996.

[6] M.C. Porcu, "Rigid-plastic inertia limiters at floor level for seismic vibration control of buildings", to appear.

[7] M.C. Porcu, "Reducing seismic stress on buildings through inertia limiters at floor level", in WIT Transactions on the Built Environment, Vol. 132, pp. 333-344, WIT Press, UK, 2013. Doi: 10.2495/ERES130271.

[8] M.C. Porcu, "Numerical Assessment of a Stress Control Method based on Rigid-Plastic Inertia-Limiters", in B.H.V. Topping, P. Iványi, (Editors), "Proc. of the XIV Int. Conf. on Civil, Struct. and Environm. Eng. Computing", Civil-Comp Press, Stirlingshire, UK, Paper 42, 2013. doi:10.4203/ccp.102.42.

[9] A. Paglietti, M.C. Porcu, Rigid-Plastic Approximation to Predict Plastic Motion under Strong Earthquakes, J. Earth. Eng. \& Struct. Dynamics, vol. 30, pp.115-126, 2001.

[10] Eurocode 8, Design provisions for earthquake resistance of structures. European Committee for standardisation, ENV 1998-1. 